\title{
THE UPGRADE OF THE ATLAS FIRST LEVEL CALORIMETER TRIGGER
}

Shimpei Yamamoto (ICEPP/UTokyo)

on behalf of the ATLAS Collaboration 


\section{Outline}

Introduction

ATLAS Liquid Argon calorimeter and level-1 trigger

$\Rightarrow$ Challenges toward high luminosity runs

OUpgrading ATLAS level-1 calorimeter trigger

$\geqslant$ New Liquid Argon calorimeter trigger readout

$\geqslant$ New system architecture for trigger processing

Expected performances

Summary 


\section{ATLAS Calorimetry}
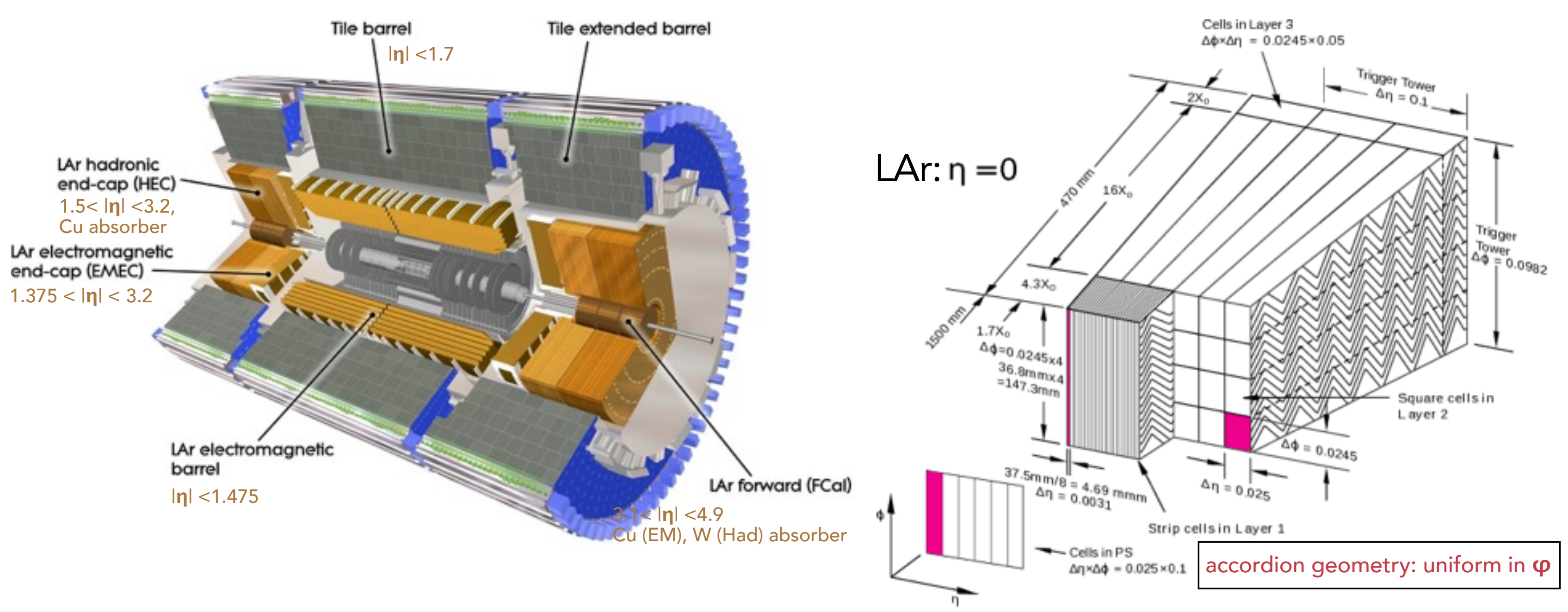

\section{Liquid Argon (LAr) electromagnetic calorimeter}

- Lead absorber, LAr as active material and copper/kapton electrode

- Has fine segmentation: 4 layers, $200 \mathrm{k}$ readout channels in total

Hadronic calorimeters : Tile (steel\&scintillator), LAr endcap/forward

$\Rightarrow$ Plays a major role in identifying/measuring $e, \gamma, T$, jet and missing $E_{T}$ (offline) and provides inputs for their triggers at the hardware level (L1Calo triggers)

- Higgs discovery! Lots of searches in variety of channels, precise measurements, .. 


\section{LHC and ATLAS trigger upgrade timelines}

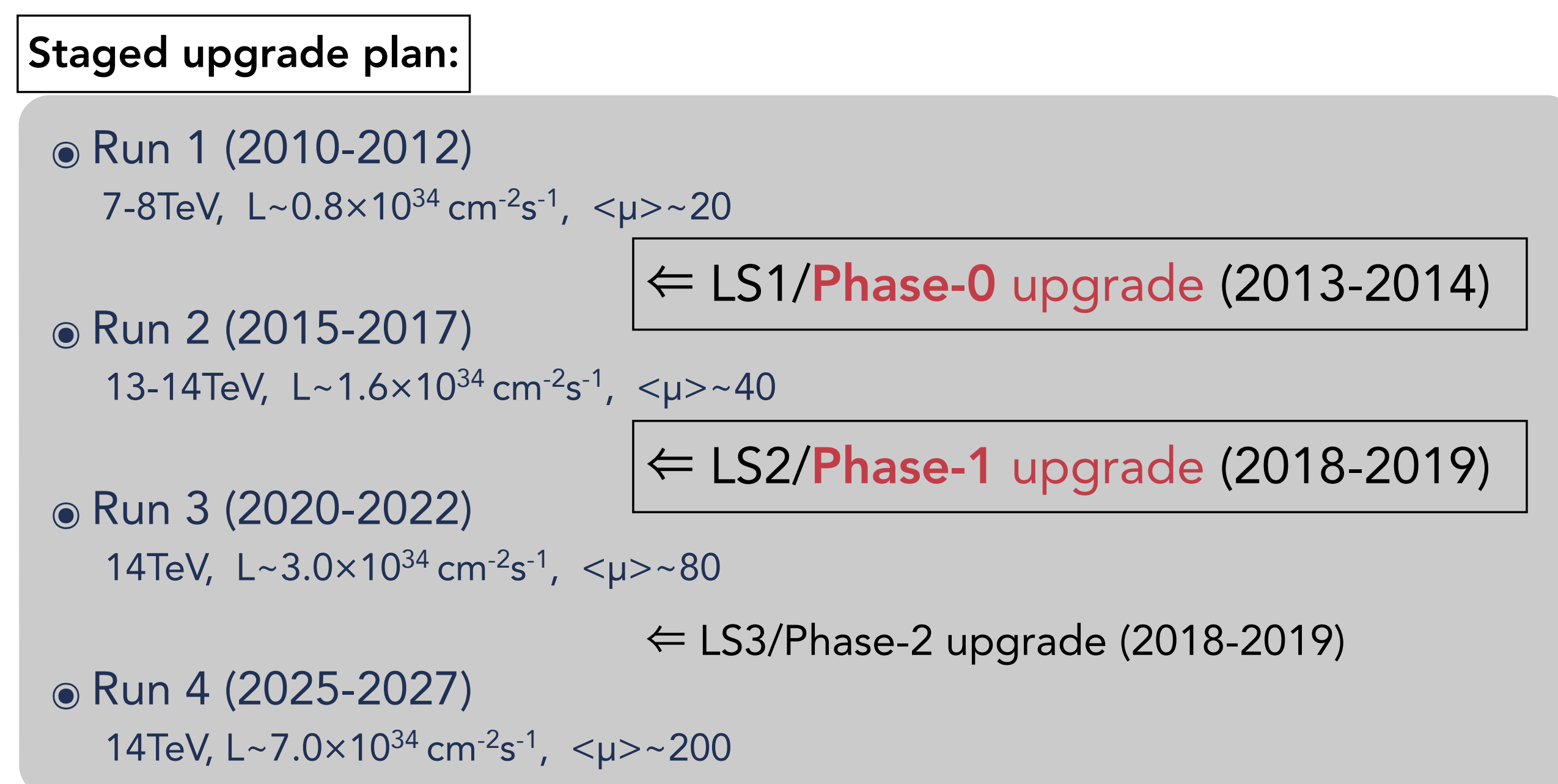

Instantaneous luminosity getting increased!!

- Number of interactions per bunch crossing $(\mu)$ doubled, tripled and much more..

"Pile-up effects" significantly degrade the trigger performance:

- Degraded LAr signal due to its long drift time: $\sim 450 \mathrm{~ns}$ drift time (with $2 \mathrm{~mm}$ gap at 2 $\mathrm{kV})$ vs. $25 \mathrm{~ns} \mathrm{LHC}$ bunch spacing $(40 \mathrm{MHz})$

- Triggering electromagnetic objects suffers from huge multi-jets background 


\section{General upgrade concept}

Utilize more calorimeter shower shape information\&event topology as in software algorithms - high-level triggers use this information as well.

$$
\begin{aligned}
& \text { e.g. e/jet separation } \\
& R_{\eta}=\frac{E_{\mathrm{T}, \Delta \eta \times \Delta \phi=0.075 \times 0.2}^{(2)}}{E_{\mathrm{T}, \Delta \eta \times \Delta \phi=0.175 \times 0.2}^{(2)}} \\
& f_{3}=\frac{E_{\mathrm{T}, \Delta \eta \times \Delta \phi=0.2 \times 0.2}^{(3)}}{E_{\mathrm{T}, \Delta \eta \times \Delta \phi=0.075 \times 0.2}^{(1)}+E_{\mathrm{T}, \Delta \eta \times \Delta \phi=0.075 \times 0.2}^{(2)}+E_{\mathrm{T}, \Delta \eta \times \Delta \phi=0.2 \times 0.2}^{(3)}}
\end{aligned}
$$

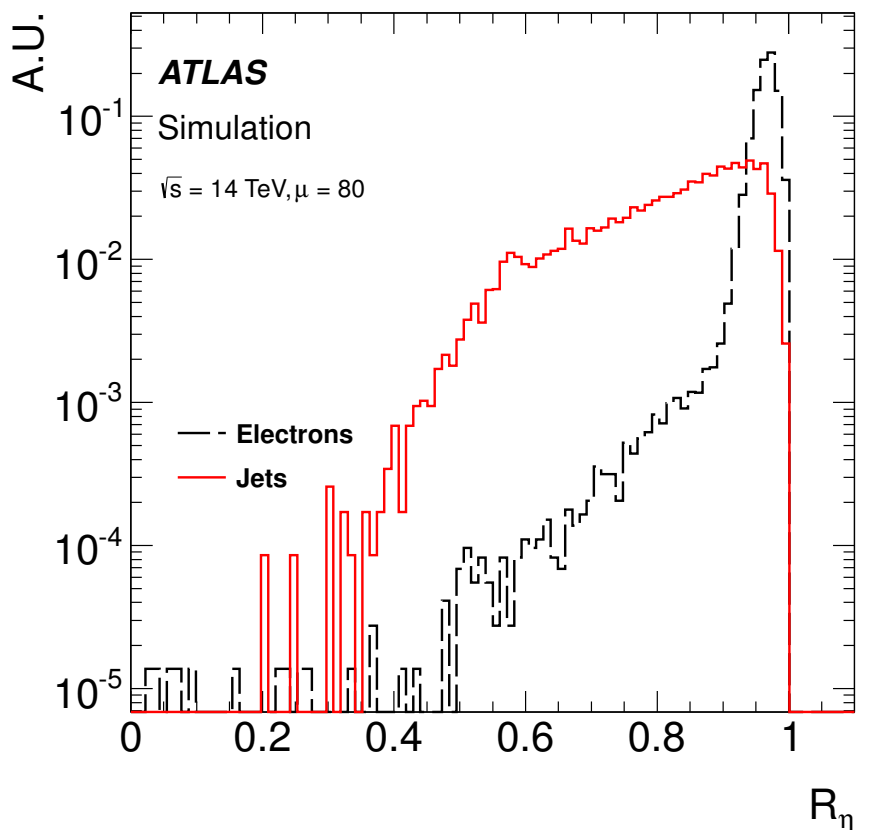

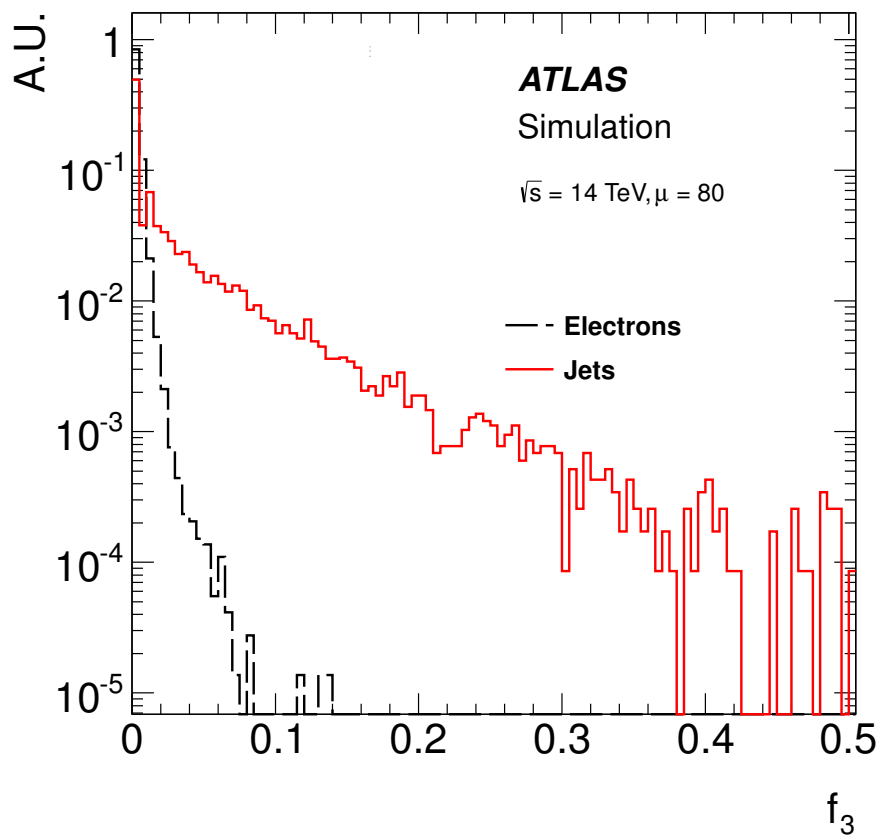

Already partially implemented in Run-2: L1Calo provides trigger capabilities based on event topologies.

- New module "L1Topo" installed. Can apply selection with topological variables as $\Delta \varphi, \Delta \eta, \Delta R, H_{T}, \ldots$
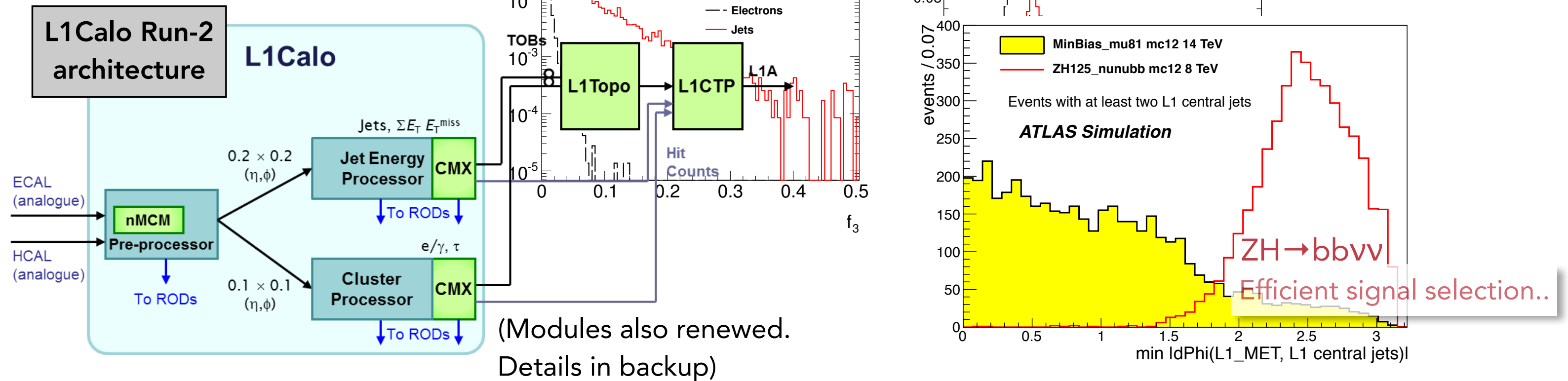

Details in backup) 


\section{General upgrade concept (2)}

To achieve these trigger features, develop new hardware for trigger readout and processing.

- LAr trigger readout

- Fully digitized readout with finer granularity \& digital filtering for out-of-time pile-up correction and bunch-crossing identification

- L1Calo trigger processing

- Pile-up subtraction, employ selection fully based on object features and event topology information

Challenges:

- Highly dense electrical\&optical circuit boards \& high-speed optical links (up to $\sim 10 \mathrm{~Gb} / \mathrm{s}$ )

- Signal mapping and data duplication among readout modules 


\section{Upgrading LAr trigger readout: Super Cells}

\section{Trigger tower}

Analog sum in $\Delta \eta \times \Delta \varphi=0.1 \times 0.1$ ( 60 cells)

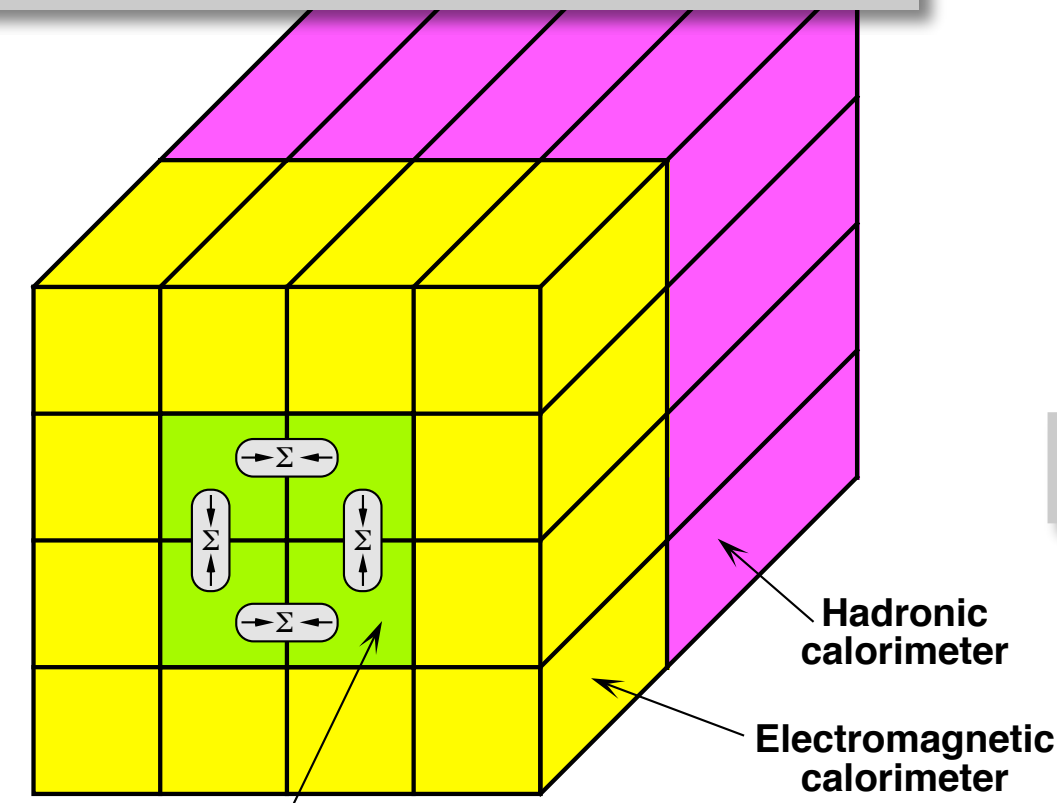

Trigger towers $(\Delta \eta \times \Delta \phi=0.1 \times 0.1)$

$\left[\begin{array}{l}1 \\ \Sigma \\ 4\end{array}\right]$ Vertical sums

$-\Sigma \oplus$ Horizontal sums Local maximum/

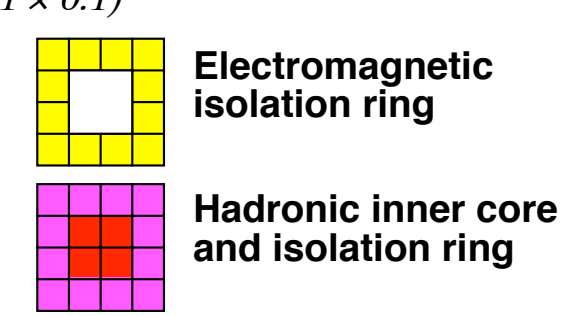

\section{Run-2 readout geometry}

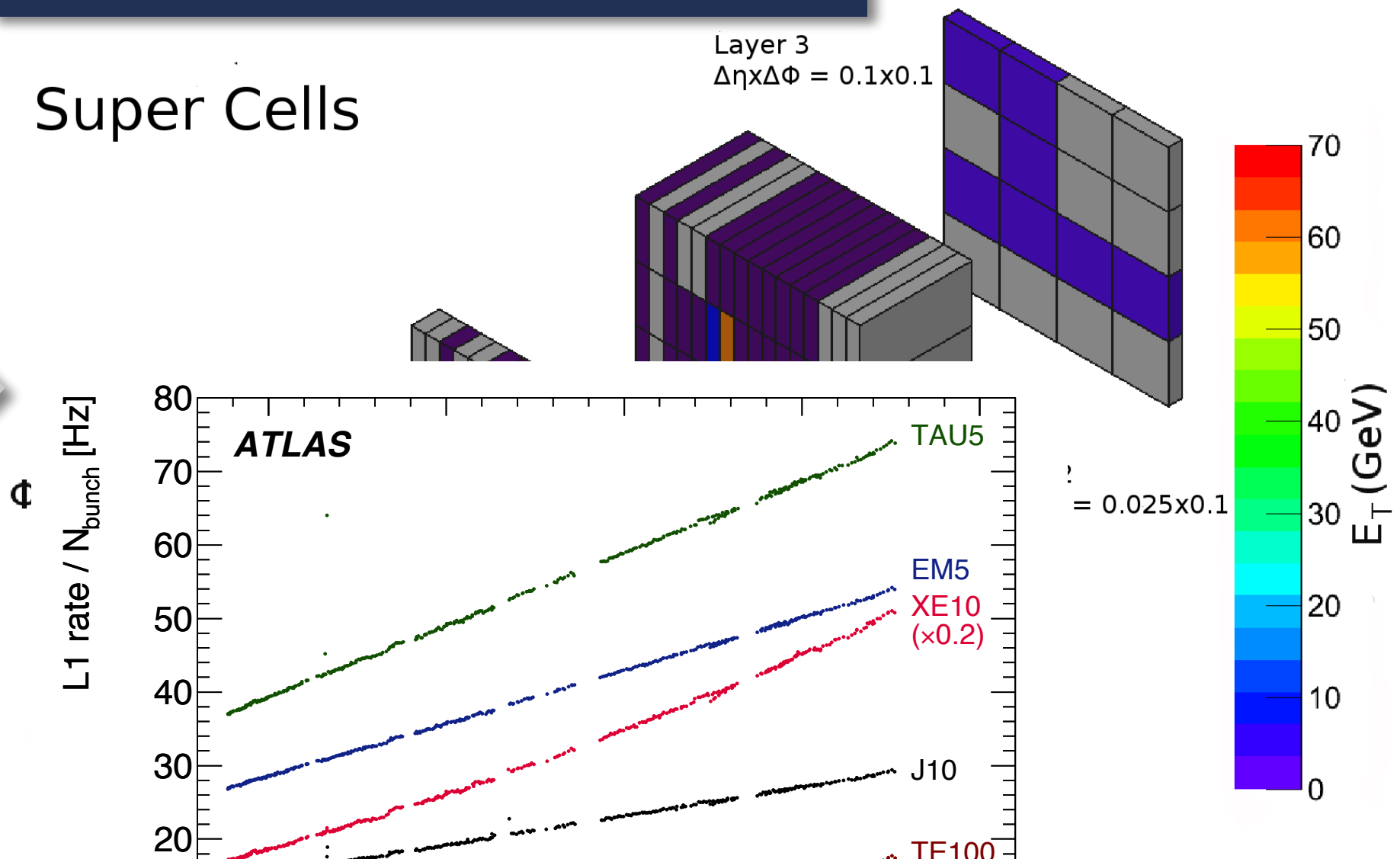

(box: minimal readout element)

$\Rightarrow$ Finer granularity: trigger tower $\Rightarrow 10$ Super Cells (SCs)

- 60 cells $\Longrightarrow 4-8$ cells in each layer

$\geqslant$ Analog readout $\Rightarrow 40 \mathrm{MHz}$ digital readout 


\section{Upgrading LAr trigger readout: Scheme}

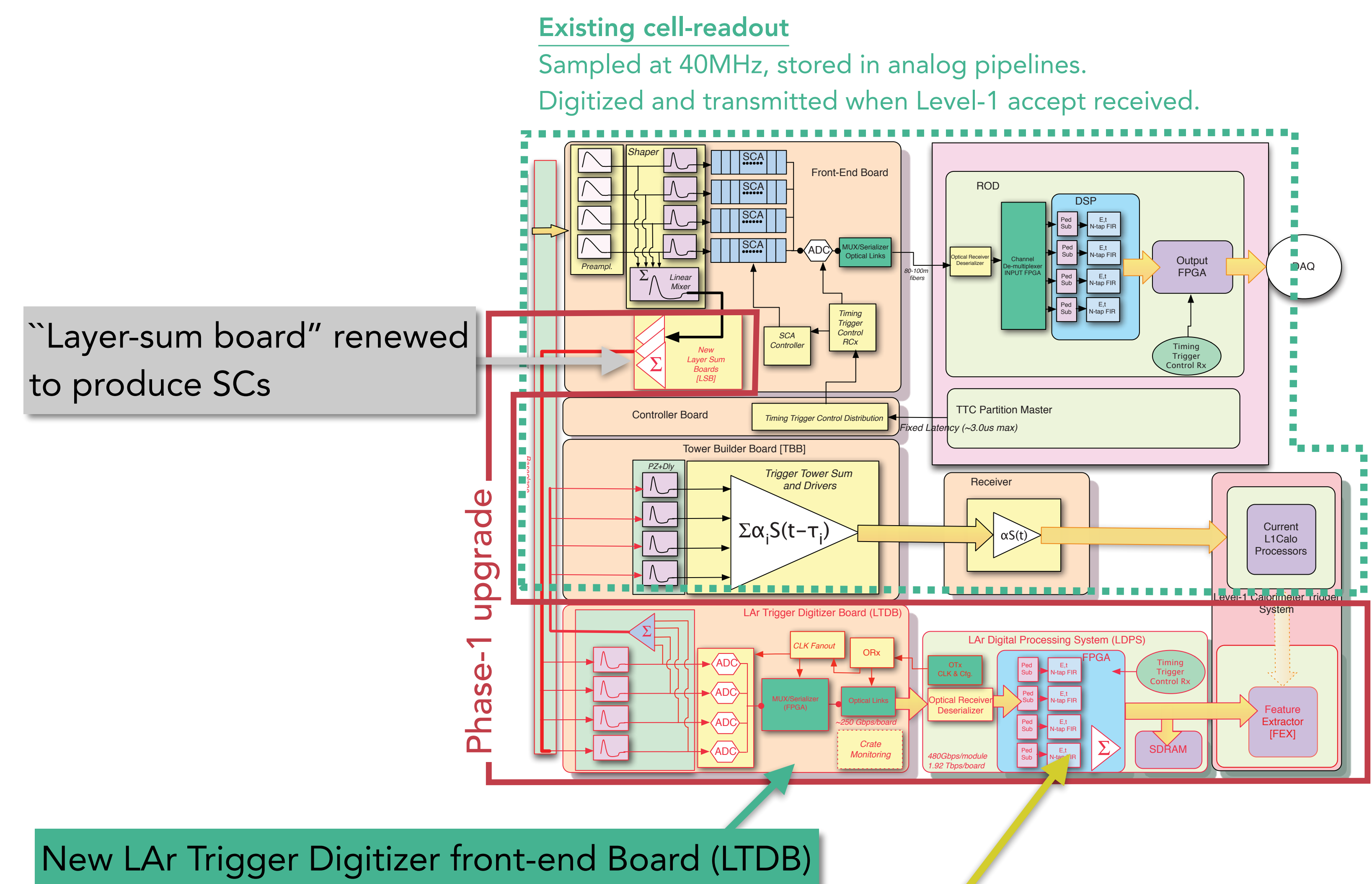

New LAr Digital processing back-end Board (LDPB) 


\section{LTDB \& LDPB}

To Tower Builder Board
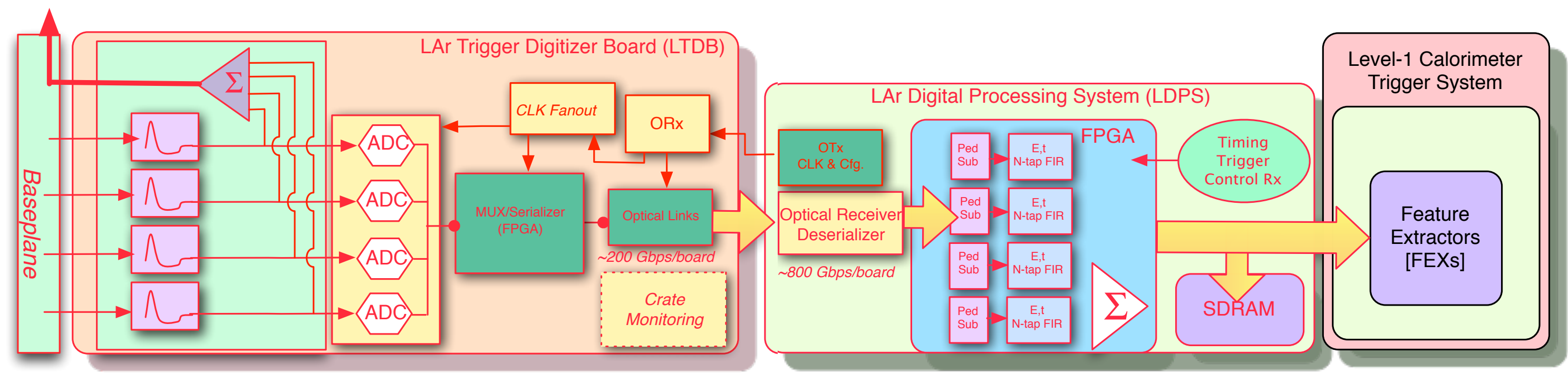

\section{LTDB}

- 12-bit ADC (custom ASIC) @40MHz

- Multiplexing 8 channels to $5.12 \mathrm{~Gb} / \mathrm{s}$ optical link, 200Gb/s for each board

- Handle up to 320 SC signals

- 124 boards in total

\section{LDPB}

- ATCA standard, 4 Advanced Mezzanine Cards (AMCs) for each

- AMC:

- high-speed optical transceivers to process 320 SCs with a short latency

- Energy\&timing measurements by FPGA digital filtering

Both LTDB and LDPS prototypes integrated in the detector system and being demonstrated during Run-2 running. 


\section{LTDB \& LDPB}

To Tower Builder Board
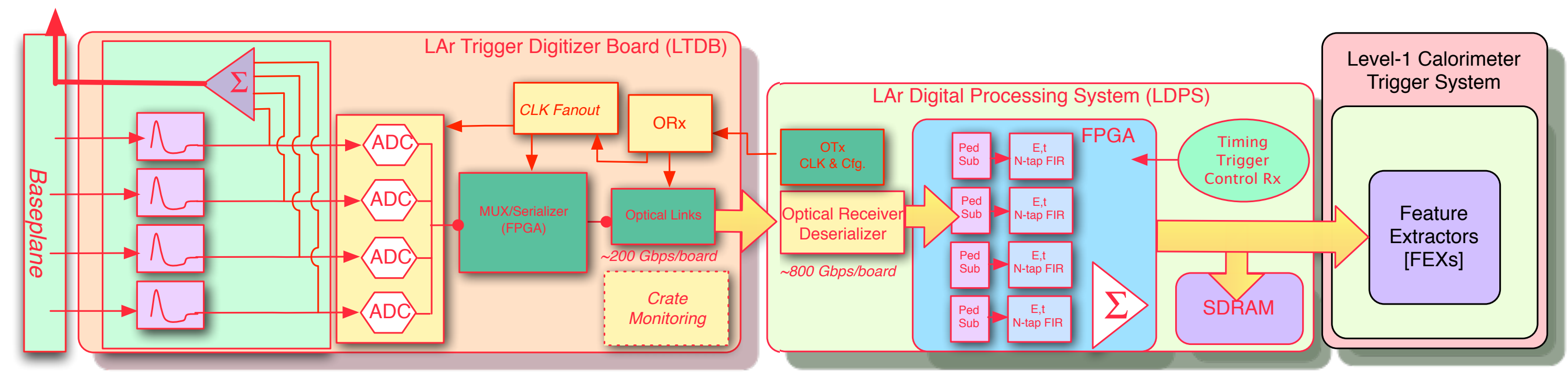

\section{LTDB}

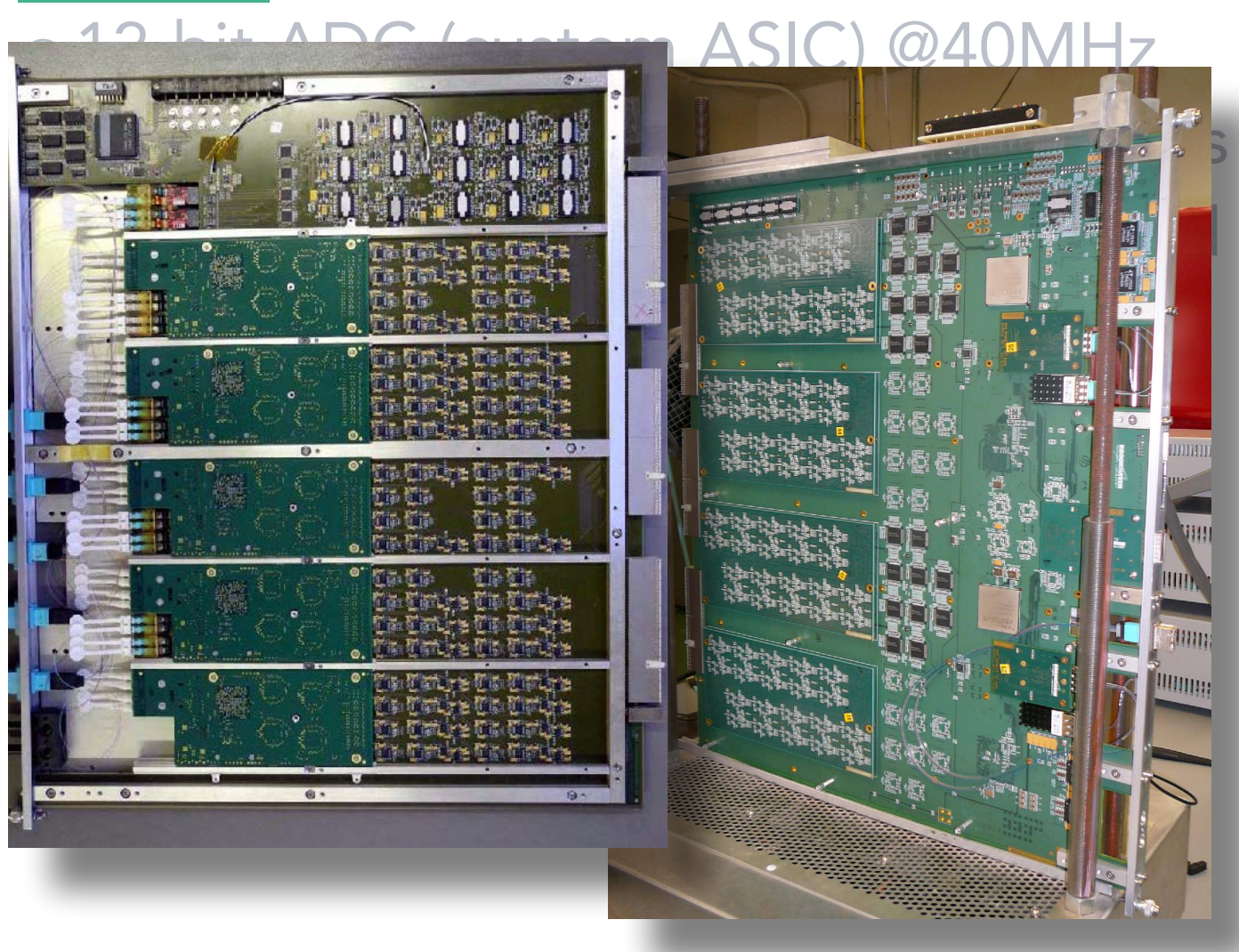

\section{LDPB}

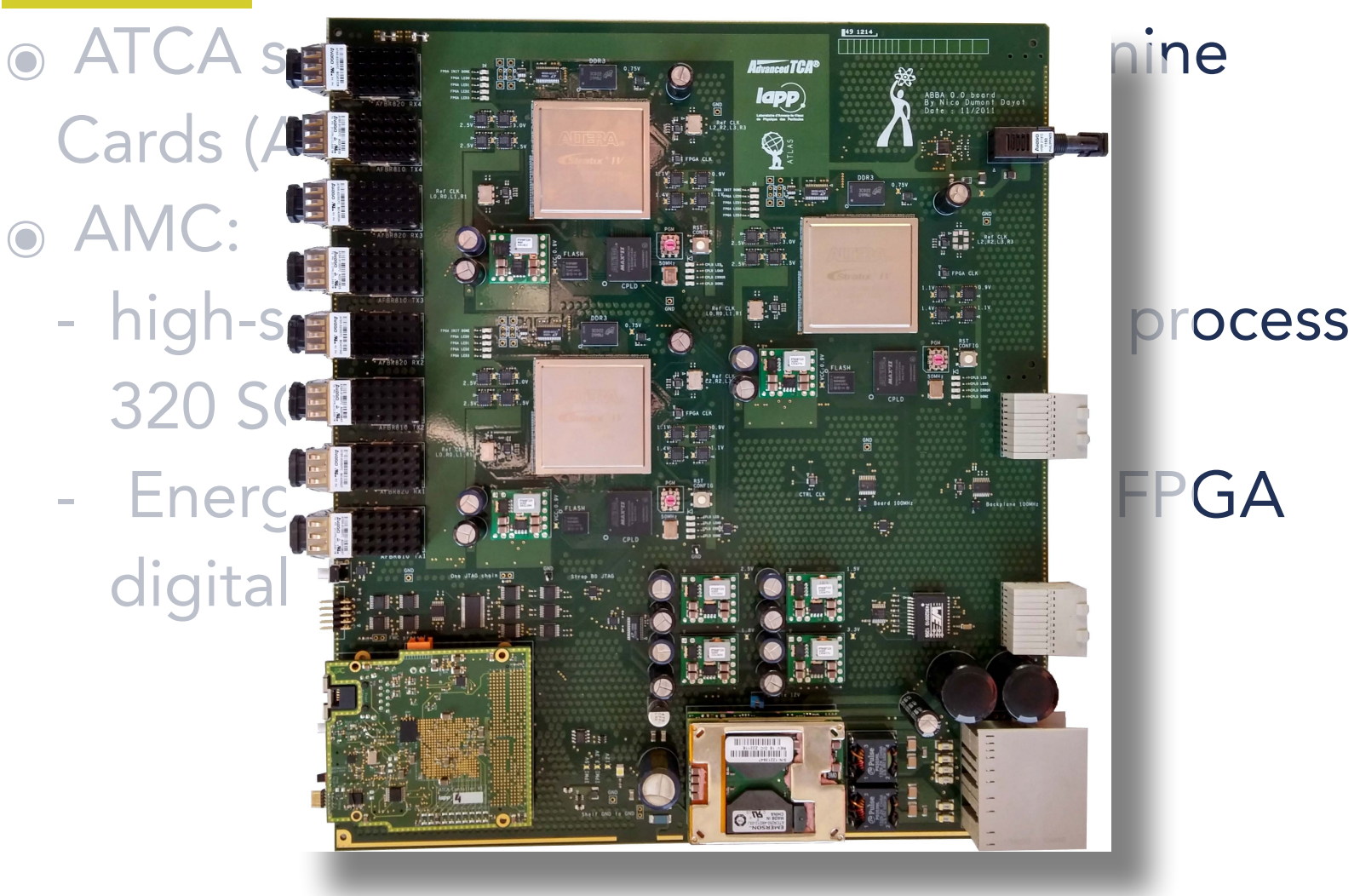

Both LTDB and LDPS prototypes integrated in the detector system and being demonstrated during Run-2 running. 


\section{SC signal reconstruction by digital filtering}

$\geqslant$ LDPB can enhance performance on the SC energy reconstruction by processing $40 \mathrm{MHz}$ ADC samplings with dedicated digital filtering.

$\Rightarrow$ Filtering algorithms under study.

e.g. Wiener filter:

- Nice "energy reconstruction" and "bunch-crossing identification"

- Expected to be pile-up robust by adopting an active forward pile-up correction

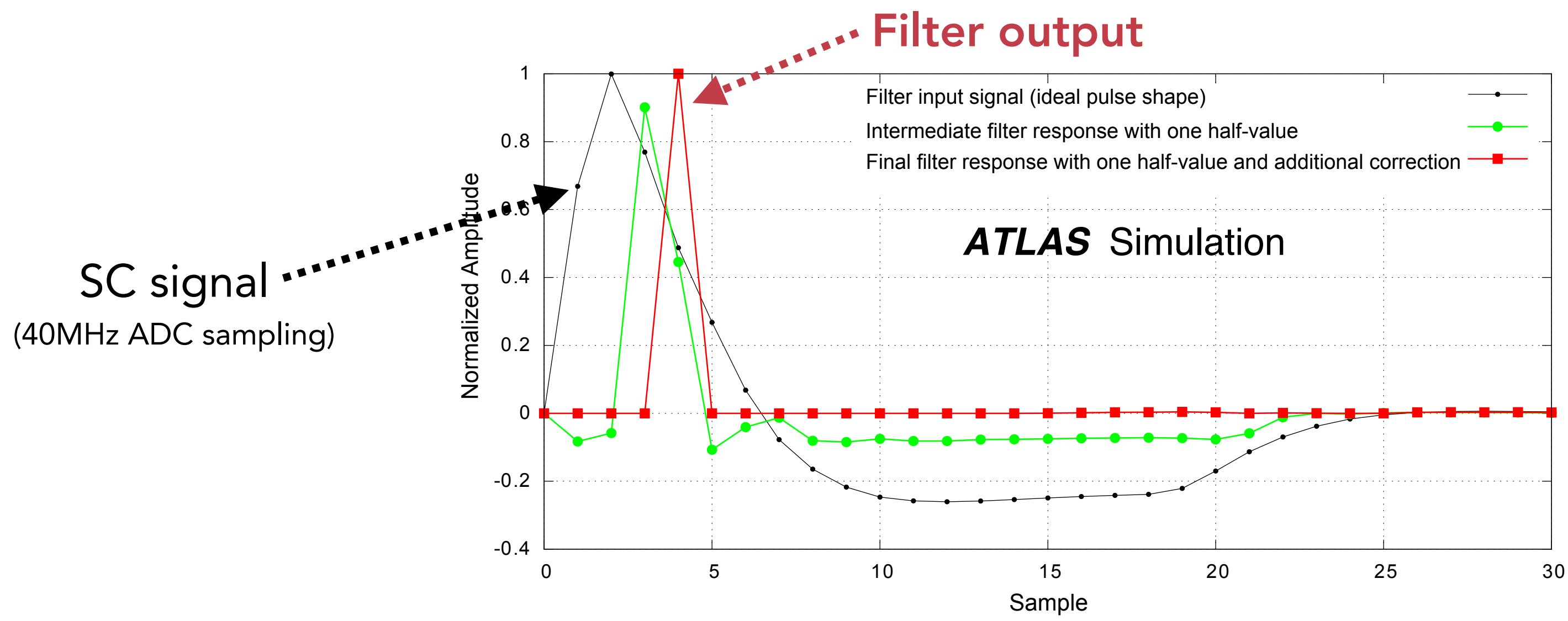




\section{Upgraded L1Calo: System architecture}
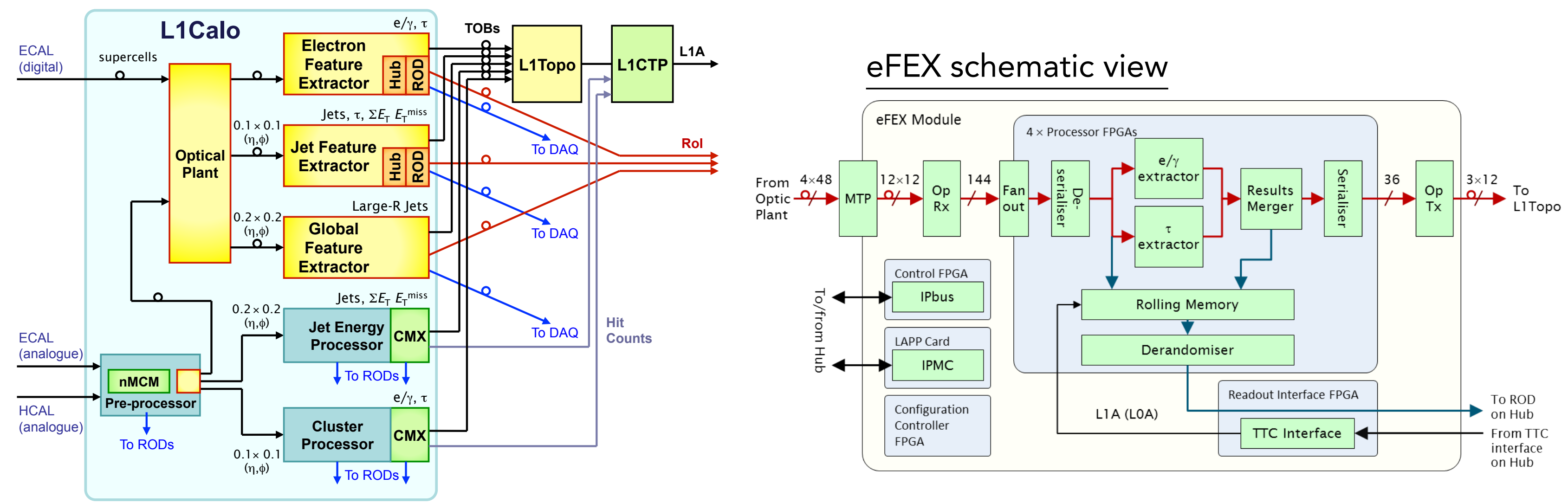

Feature extractor modules integrated for Run-3.

eFEX:

Identify $\mathrm{e} / \mathrm{Y} / \mathrm{T}$ using isolation \& cluster shape variables. Flexible rejection algorithms. jFEX:

Identify jet/T and calculate $H_{T}$, missing $E_{T}$.

Enables pile-up suppression using event energy density

gFEX:

For global event processing, e.g. large-area jets for dedicated physics cases (boosted bosons, ...) 


\section{Expected performance: single-object triggers}

\section{$\geqslant$ EM trigger rate:}

- Adopting jet rejection using shape variables, the threshold can be lowered by $7 \mathrm{GeV}$

- Compared at reference points of $20 \mathrm{kHz}$ (Run-2 rate budget) and $95 \%$ efficiency

- Can maintain high photon efficiency (>96\%)

Better trigger turn-on for jet and missing $\mathrm{E}_{\mathrm{T}}$ triggers

- Thanks to pileup suppression and dedicated jet reconstruction algorithm
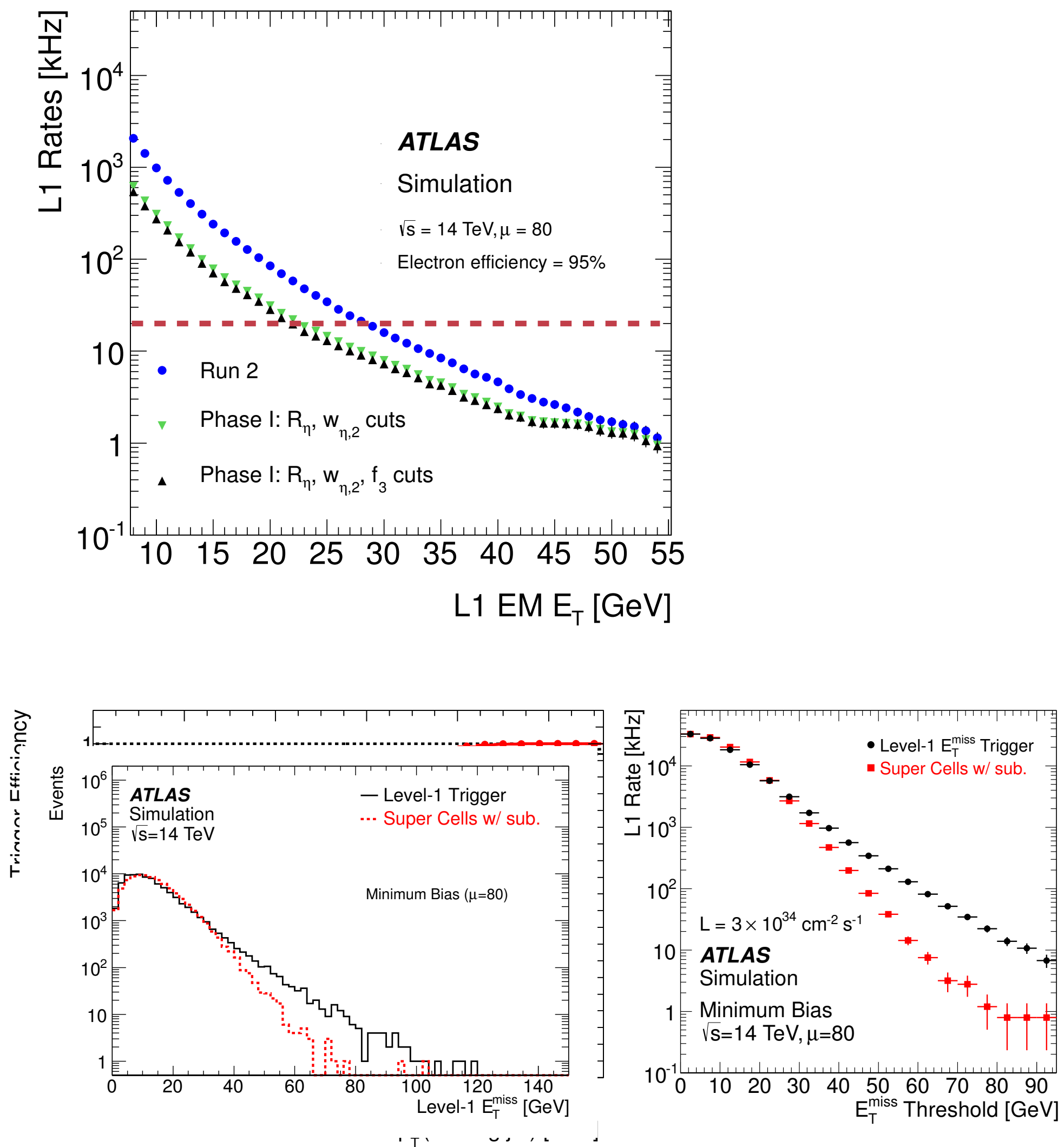


\section{Summary}

D Upgrade activities ongoing in order to explore full physics of the high-luminosity LHC runs.

ATLAS is developing new hardware and system for calorimeter trigger readout and processing.

- Expect improved trigger performances with the upgraded system even in severe pile-up conditions.

- R\&D, design and production in progress toward installation in 2018-2019 (Phase-1 upgrade)

- Some of them are already integrated for ATLAS Run-2 running:

- LTDB \& LDPS prototypes for demonstration

- Topological trigger with new L1Calo architectures 


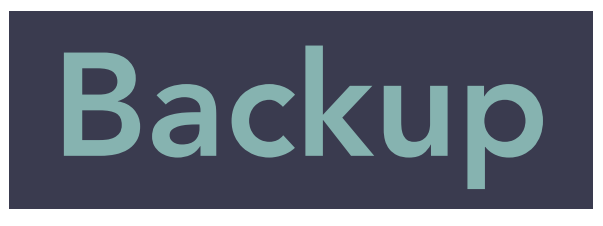




\section{Super Cells}

\section{Trigger tower $\Rightarrow 10$ Super Cells (SCs)}

Trigger towers

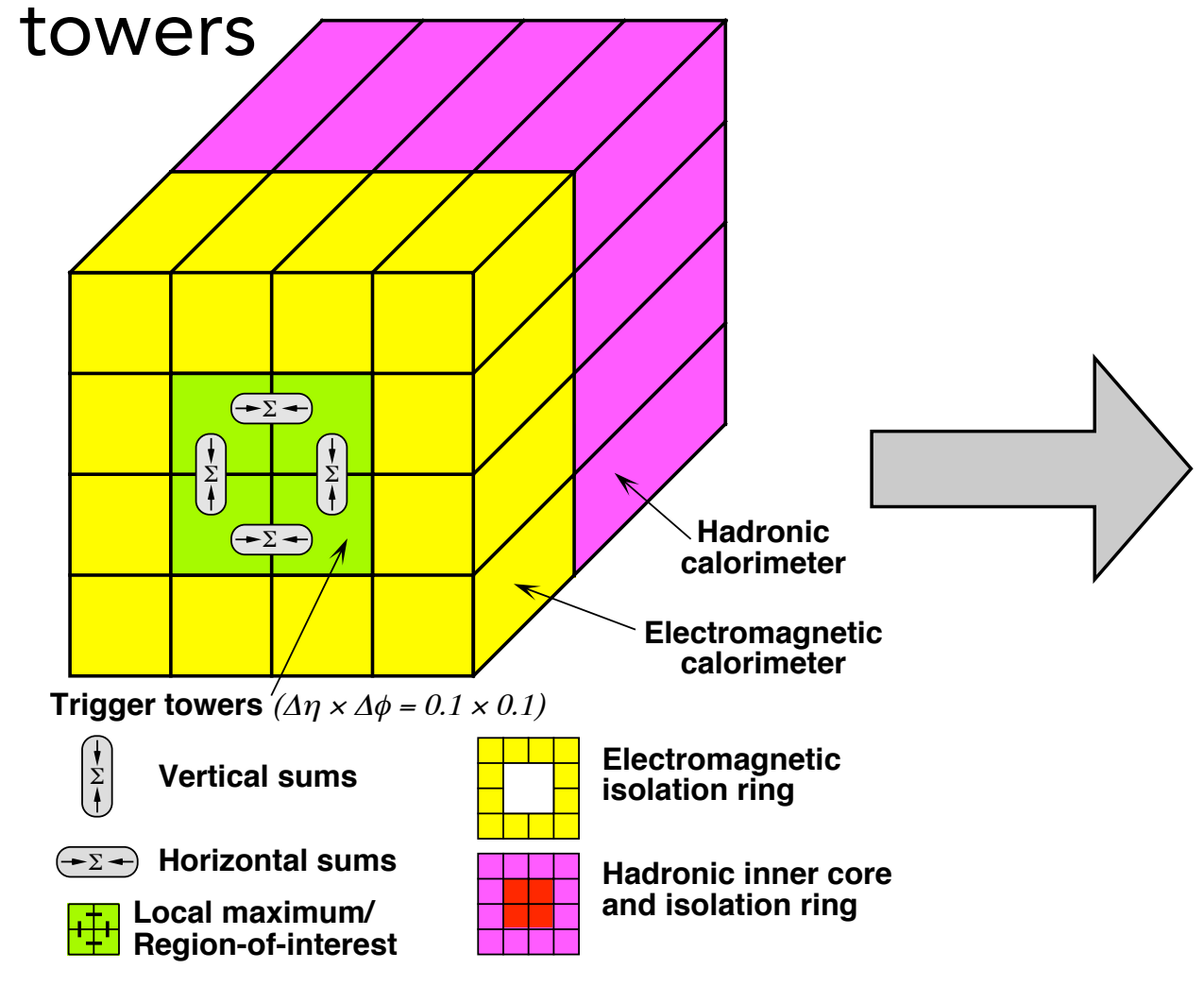

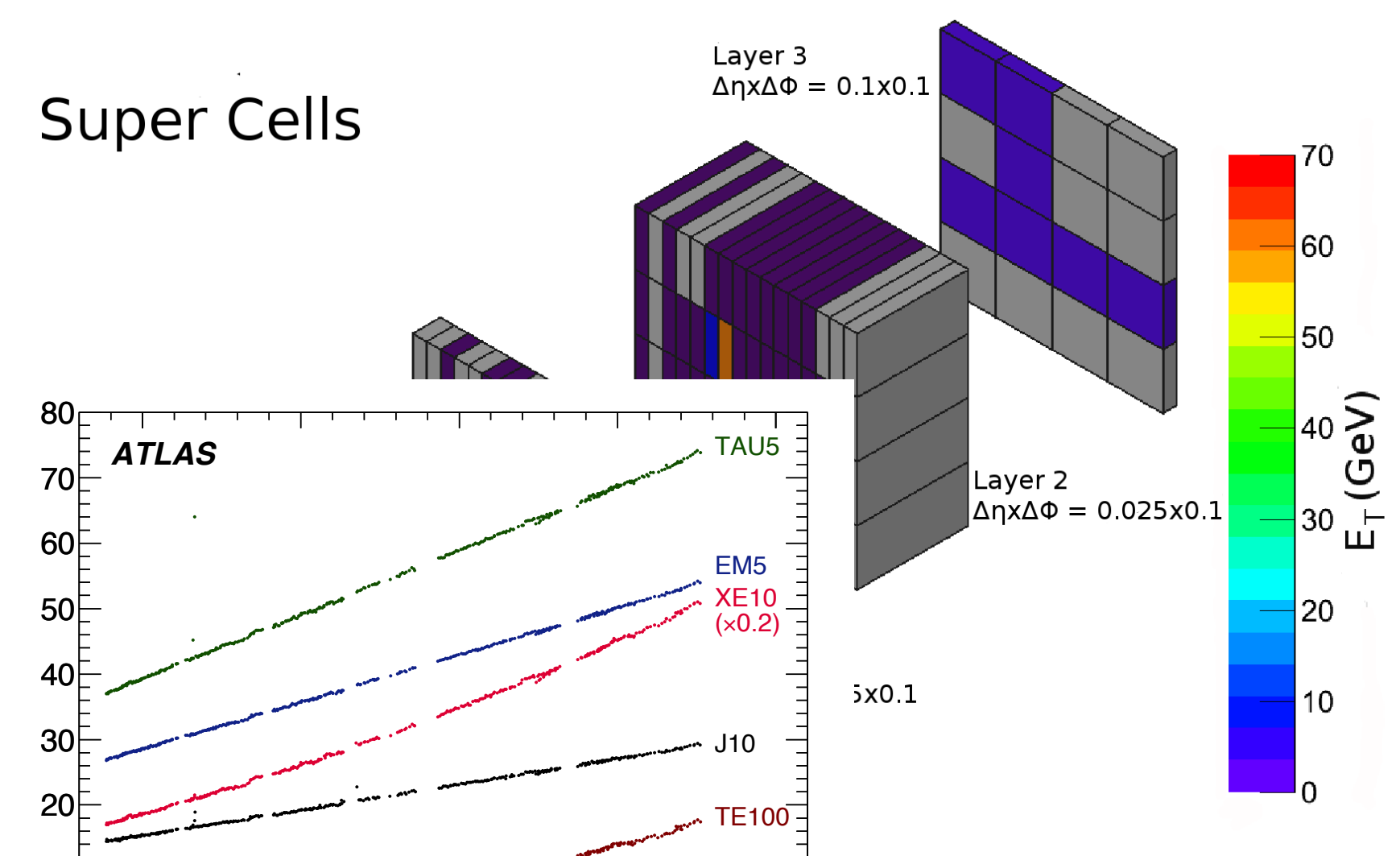

\begin{tabular}{c|c|c|c|c|c} 
& \multicolumn{2}{c}{ Elementary Cell } & \multicolumn{2}{c}{ Trigger Tower } & \multicolumn{2}{c}{ Super Cell } \\
\hline Layer (barrel) & {$[\Delta \eta \times \Delta \varphi]$} & {$\left[\mathrm{n}_{\eta} \times \mathrm{n}_{\varphi}\right]$} & {$[\Delta \eta \times \Delta \varphi]$} & {$\left[\mathrm{n}_{\eta} \times \mathrm{n}_{\varphi}\right]$} & {$[\Delta \eta \times \Delta \varphi]$} \\
\cline { 2 - 3 } Presampler (layer 0) & $0.025 \times 0.1$ & $4 \times 1$ & & $4 \times 1$ & $0.1 \times 0.1$ \\
\cline { 2 - 3 } Front (layer 1) & $0.003125 \times 0.1$ & $32 \times 1$ & \multirow{3}{*}{$0.1 \times 0.1$} & $8 \times 1$ & $0.025 \times 0.1$ \\
\cline { 2 - 3 } \cline { 5 - 6 } Middle (layer 2) & $0.025 \times 0.025$ & $4 \times 4$ & & $1 \times 4$ & $0.025 \times 0.1$ \\
\cline { 2 - 3 } Back (layer 3) & $0.05 \times 0.025$ & $2 \times 4$ & & $2 \times 4$ & $0.1 \times 0.1$
\end{tabular}




\section{LTDB \& LDPB specifications}

\section{To Tower Builder Board}

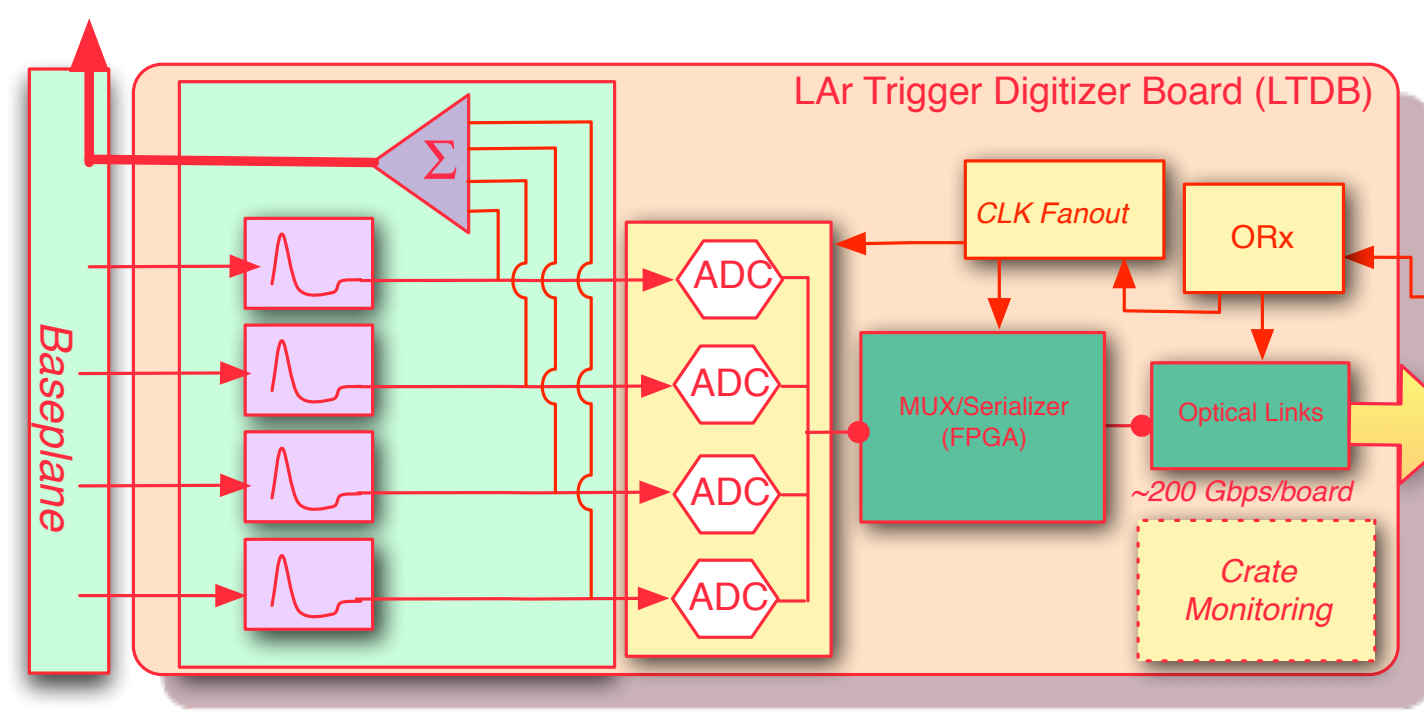

\section{LTDB}

- Handle up to 320 SC signals

- 12-bit ADC @40MHz with low power consumption (custom ASIC)

- <145 mW/channel

- Multiplexing 8 channels to $5.12 \mathrm{~Gb} / \mathrm{s}$ optical link, 200Gb/s for each board

- 124 boards in total (25Tb/s)

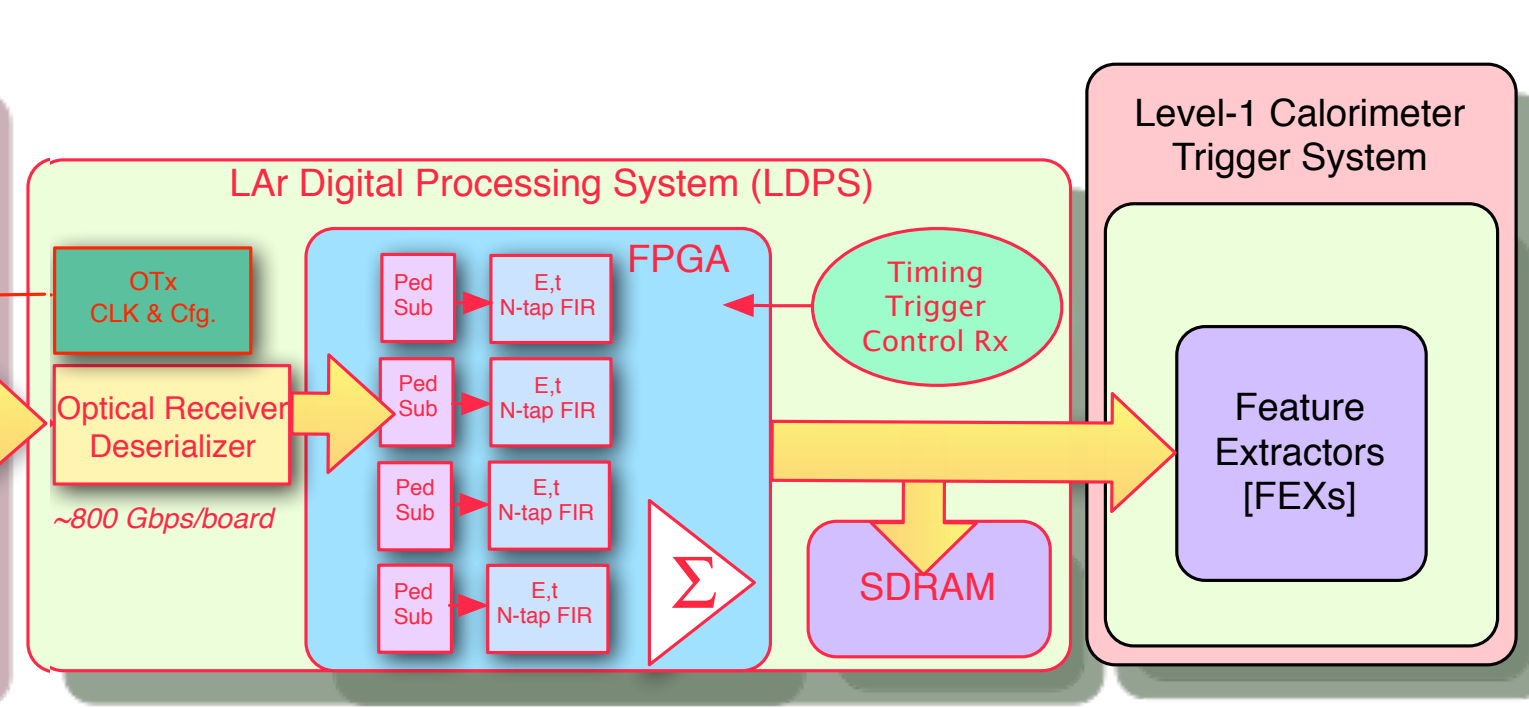

\section{LDPS}

- ATCA standard, 4 Advanced Mezzanine Cards (AMCs) for each

- AMC designed with FPGA and high-speed optical transceivers to process 320 SCs with a required latency (17 bunch crossings)

- Energy measurement\&bunch-crossing identification by FPGA digital filtering Receive 25Tb/s (from LTDB) and transmit $41 \mathrm{~Tb} / \mathrm{s}$ (to L1Calo) 


\section{Upgraded L1Calo: Run-2 system architecture}

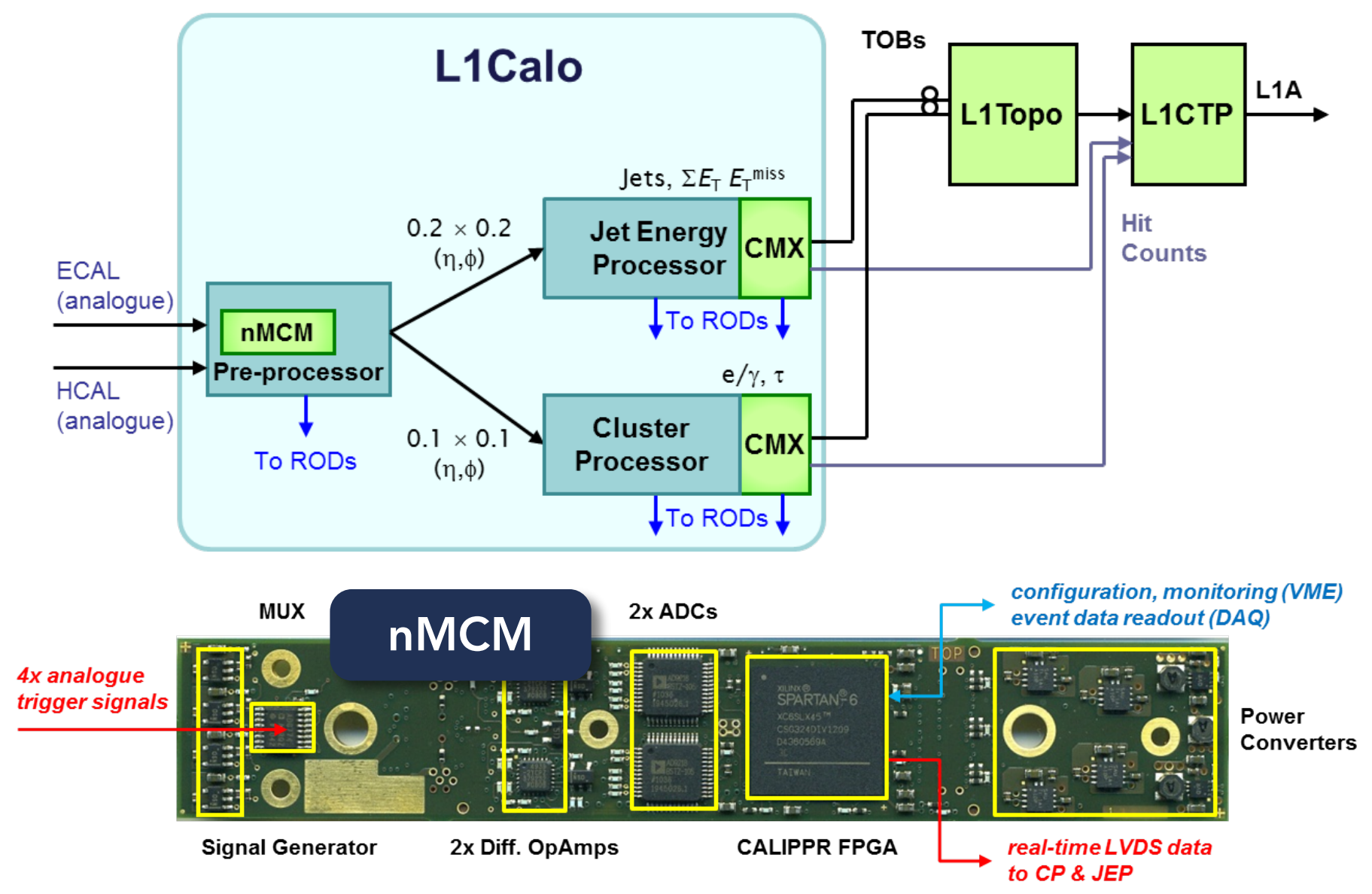

Phase-0 upgrade (for Run 2)

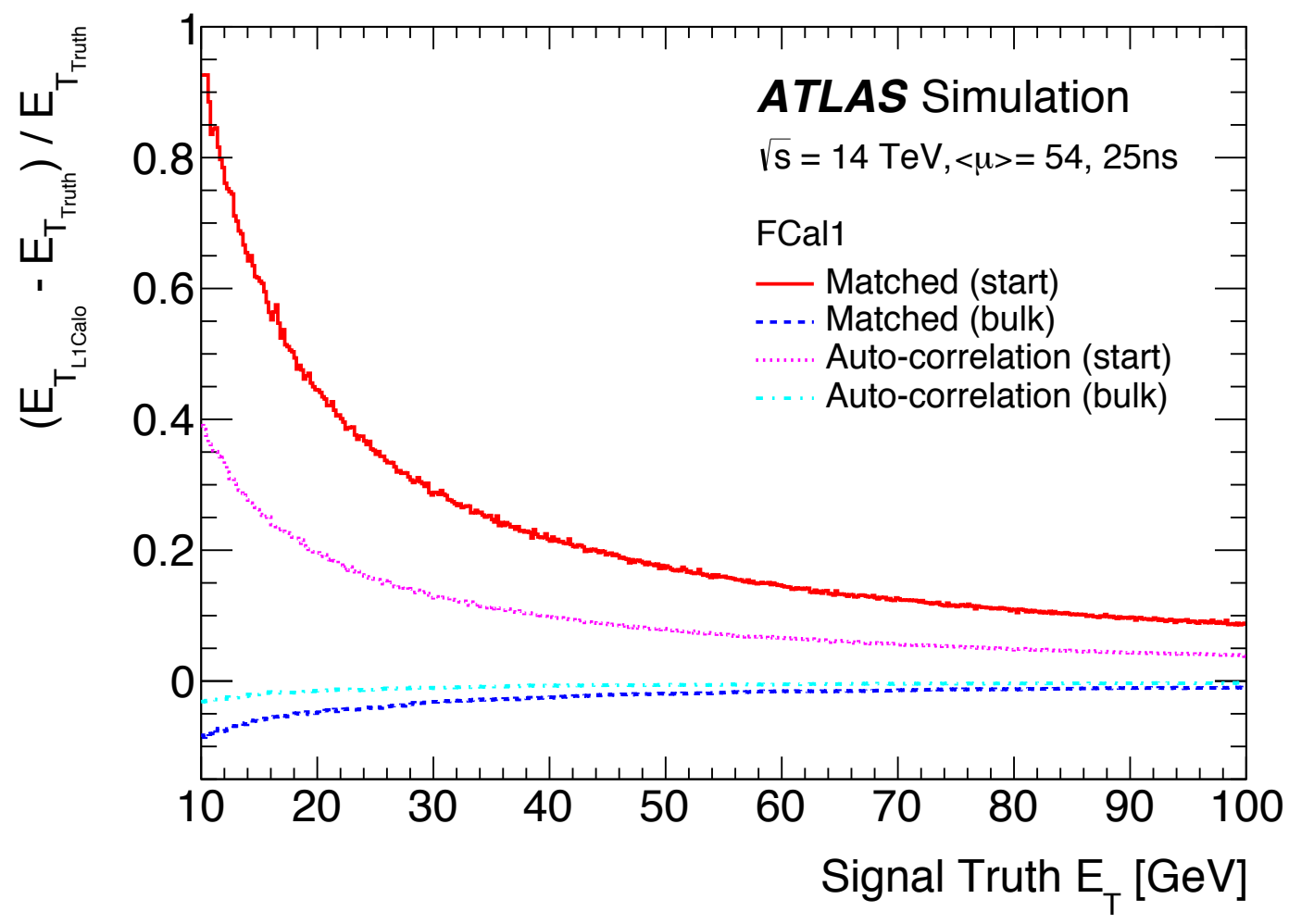

New Multi Chip module (nMCM):

ASIC $\rightarrow$ FPGA replacement for digital signal processing

Better treatment of pile-up (autocorrelation FIR filter \& dynamic pedestal subtraction)

Dedicated calibration for EM and hadron energy scale using dual-channel ADCs

New(extended) Common Merger Module (CMX):

$160 \mathrm{Mb} / \mathrm{s}$ backplane (data from processor modules), multiplicities $\rightarrow$ trigger objects.

Link to L1Topo (24 opt. fibers at $6.4 \mathrm{~Gb} / \mathrm{s}$ )

Topological trigger module (L1Topo):

Enables composite triggers using trigger objects. Topological algorithms. 


\section{Expected performance: L1Topo, FEX}

\section{L1Topo (already installed for Run-2)}

- Can apply selection with topological variables: $\Delta \varphi, \Delta \eta, \Delta R, H_{T}, \ldots$

- Challenging analyses will benefit from that.

- ZH $\rightarrow$ vvbb, di-tau etc.

\section{jFEX (Run-3)}

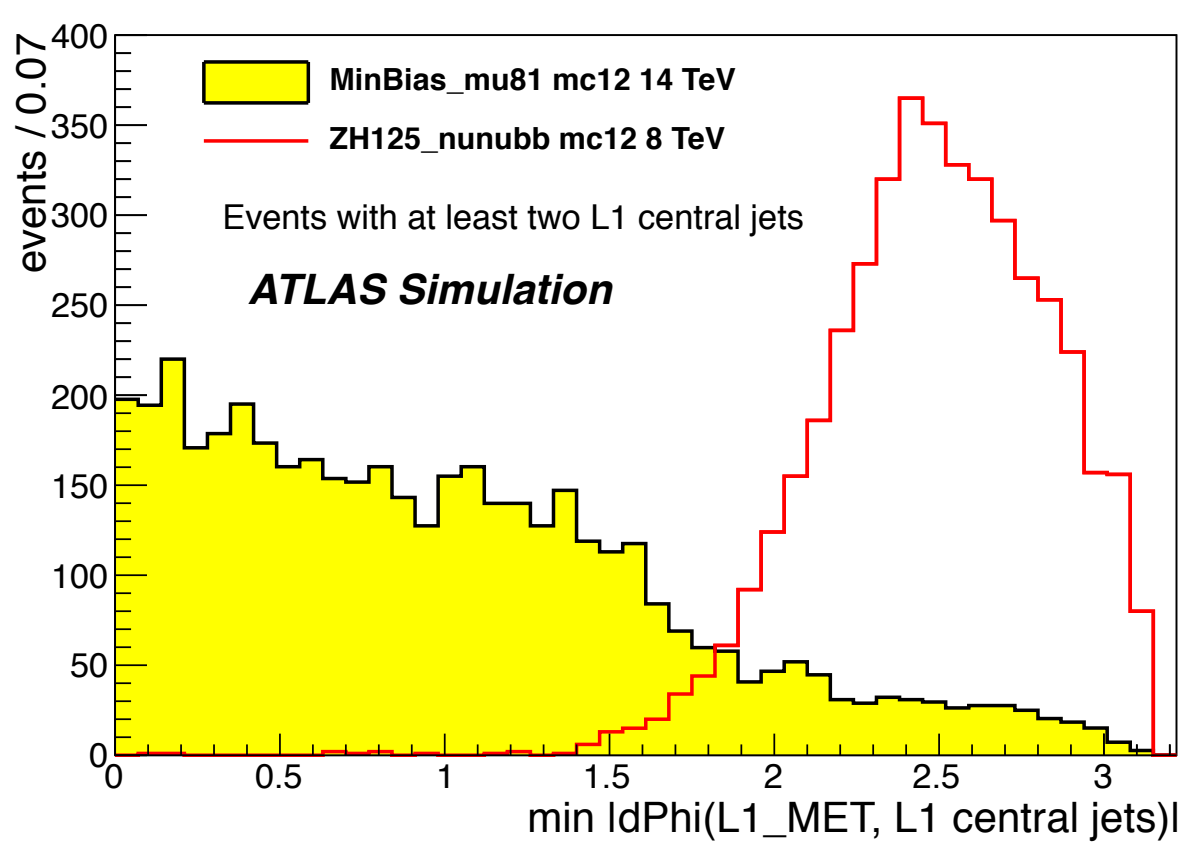

- Gaussian filter: jet energy reconstruction using Gaussian weights

- Less sensitive to pile-up, significant rate reduction thanks to improved trigger turn-on.

- Expect much better performance by adopting calibrations and optimizing in terms of pile-up.
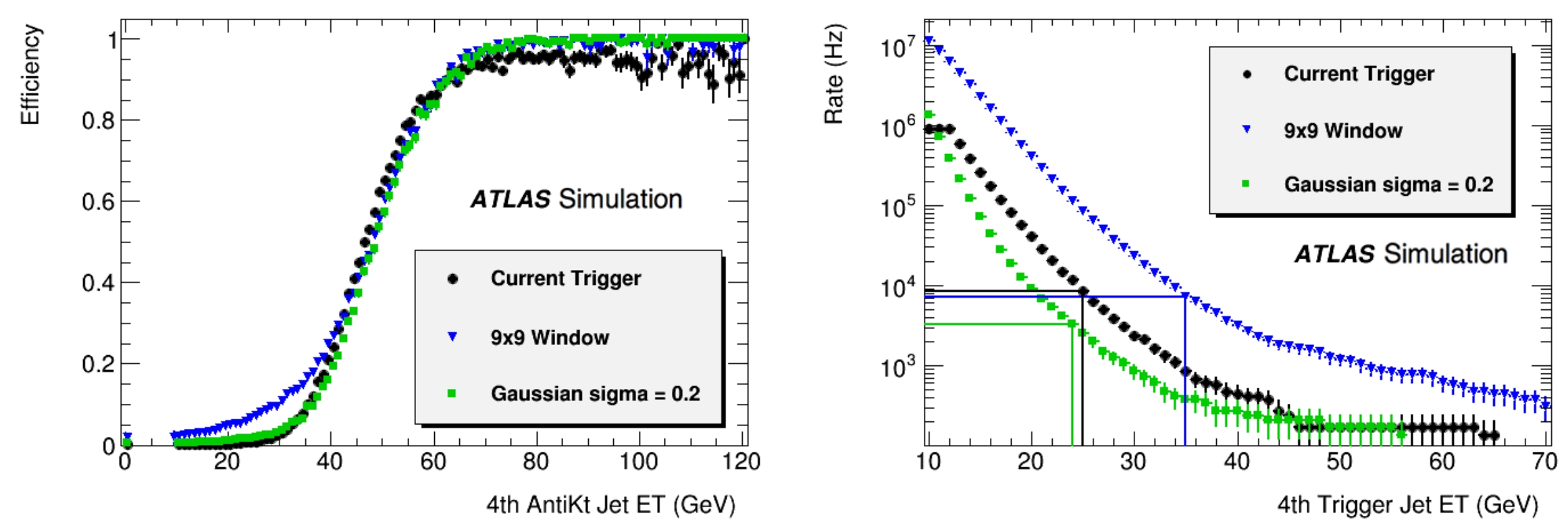